\title{
Violencia simbólica contra las mujeres en la enseñanza de la filosofía. Roles del o la docente y la escuela
}

Symbolic violence against womens in the teaching of philosophy. Roles of the teacher and the school

Asdrúbal Hernán Serna Urrea

Universidad de Antioquia, Colombia

asdrubal.serna@udea.edu.co

\section{Resumen:}

Hay un buen número de filósofas identificadas en la historia de la filosofía, sin embargo el canon no las incluye, por lo que se acusa a la filosofía de androcéntrica y misógina. Su enseñanza, puede contribuir a reproducir el paradigma según el cual el varón domina el espacio público y el campo de poder económico, en tanto las mujeres son relegadas al espacio privado o doméstico y no intervienen en la producción de saberes. Por esa razón, es importante reflexionar en torno a los roles del o la docente, la escuela, el currículo oculto y los textos escolares en el nivel secundario, y cómo todos esos elementos pueden ejercer violencia simbólica contra las mujeres, pues a través de ellos se efectúa la enseñanza. Este artículo explora este problema y arroja algunas indicaciones de lo que podría hacerse para minimizarlo: formación docente, inserción de mujeres en el canon, enseñanza en equidad de género. Palabras clave: Filósofas, Canon filosófico, Currículum oculto, Textos escolares, Equidad de género, Sexismo en la escuela.

\section{Abstract:}

There are a significant number of women philosophers identified in the history of philosophy, but the canon does not include them. So, philosophy is accused of being androcentric and misogynistic. Its teaching can contribute to perpetuation of a paradigm in which man dominates the public space and the field of economic power, while the woman is relegated to the private or domestic space and is not involved in the production of knowledge. So, it is important to consider the role of teachers, school, the 'hidden curriculum' and textbooks in secondary. Particular attention should be paid to how they can exert symbolic violence against women, because through these essential elements the teaching is carried out. This article explores this problem and provides a framework by which to curtail it. Specific areas requiring improvement are teacher training, integration of women in the canon, and gender equity.

KEYWORDS: Women philosophers, Philosophical canon, Hidden curriculum, School texts, Gender equity, Sexism in school.

\section{INTRODUCCIÓN}

Incesantemente, las mujeres son víctimas de los estereotipos de dominación masculina que atraviesan la tradición occidental (Bourdieu, 2000) ${ }^{1}$ especialmente en Latinoamérica. A pesar del rechazo casi unánime de la sociedad hacia la violencia contra las mujeres ${ }^{2}$, es necesario señalar que la dominación masculina parece trascender las voluntades de varones y mujeres, y funcionar como un sistema autónomo y sutil que sabe invisibilizar las formas de violencia simbólica. Para mostrar esta situación es útil la categoría "lógica de la denegación" expresada por Bourdieu y Saint Martin (1998, p. 8), que consiste en que dicho sistema "hace lo que hace en las formas tendientes a demostrar que no lo hace" ${ }^{\prime 3}$. En ese mismo orden de ideas, Wieviorka (2006) sugiere que la violencia simbólica no es fácil de definir porque escapa a las estadísticas.

La dominación masculina no es otra cosa que el patriarcado, y cuya definición más amplia "es la manifestación y la institucionalización del dominio masculino sobre las mujeres y las/os niños/as de la familia 
y la ampliación de ese dominio masculino sobre las mujeres a la sociedad en general” (Lerner, 1990, en Bach, 2015, p. 32)

Una de las instituciones que refuerza la dominación masculina es la escuela (Galak, 2015; Bourdieu, 2000). Las y los docentes representamos una figura que influencia y moldea estos estereotipos (Mauss, 1997). Del mismo modo, el currículo actúa como un regulador para mantener estos estereotipos y "perpetuar el poder" (Masschelein y Simons, 2014, p. 6). Adicionalmente, el currículo oculto esconde la manera en que estos estereotipos se transmiten (Spadaro y Femenías, 2012). Sin embargo, es en la misma escuela donde esperaríamos generar los cambios necesarios para visibilizar estos estereotipos y frenar su transmisión (Van Dijk, 2007; Álvarez Uría, 1995).

De las múltiples maneras a través de las cuales la dominación masculina se manifiesta en la escuela están el canon, los discursos e imágenes de los libros de texto, los gestos y el lenguaje sexista, las relaciones estudiantedocente, entre otras más sutiles y, por lo mismo, más peligrosas. Como consecuencia de esta dominación, la violencia simbólica hacia las mujeres continúa perpetuándose.

La enseñanza de la filosofía no escapa a esta acusación que recae sobre la escuela, ni siquiera a pesar de su pretendida neutralidad. ${ }^{4}$ Por el contrario, la filosofía como disciplina y como discurso ha sido una de las mayores perpetradoras de violencia contra las mujeres.

Nuestra posición, que compartimos con Da Cunha (2015), es que "toda educación debe dejar de ser androcéntrica” (p. 192). Y por ello es que los planes de estudio y las políticas educativas deben incluir cuestiones de género, algo que actualmente no ocurre con frecuencia. Lo más grave es que en el currículum nulo ${ }^{5}$ se reproducen constantemente los estereotipos de género que generan violencia simbólica.

Este artículo es un llamado de atención a los maestros y maestras de educación básica secundaria que ignoran o están desinteresadas/os en reflexionar desde su práctica pedagógica sobre los mecanismos a través de los cuales la escuela y la filosofía, como área de enseñanza, reproducen y transmiten violencia simbólica contra las mujeres. Esta violencia trasciende la ya denunciada ausencia de las mujeres en el canon filosófico porque se ubica en el núcleo mismo del ejercicio del filosofar. Además de analizar el problema, se muestra cuál puede ser el rol del o la docente en la escuela para que tal violencia disminuya.

Así, las dos primeras secciones de este texto muestran cómo es que la escuela y la filosofía reproducen estereotipos patriarcales. La tercera, y a modo de conclusión, indica el rol que docente y escuela pueden tener para enfrentar la violencia simbólica contra las mujeres producida inconscientemente.

\section{LA ESCUELA COMO REPRODUCTORA DE ESTEREOTIPOS PATRIARCALES DESDE SU CURRÍCULO}

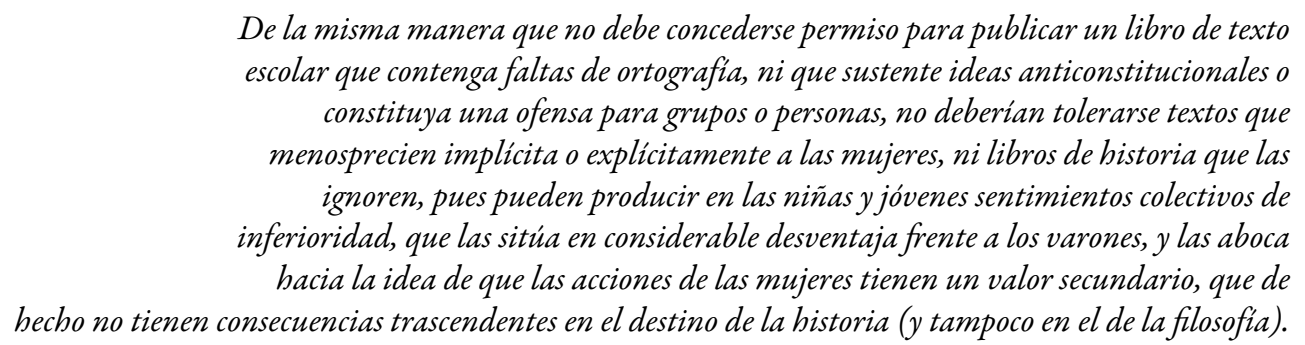

(Spadaro, 2008-2009, p. 133)

El modo en que la escuela reproduce la violencia simbólica contra las mujeres se evidencia en un análisis del currículo, tanto explícito como oculto, y específicamente de los textos escolares, así como de las relaciones entre docentes y estudiantes. Esta sección muestra cómo se presenta esa violencia. Se entiende por currículo la definición dada por De Alba (1998):

síntesis de elementos culturales (conocimientos, valores, costumbres, creencias, hábitos) que conforman una propuesta político-educativa pensada e impulsada por diversos grupos y sectores sociales cuyos intereses son diversos y contradictorios, en donde algunos de éstos son dominantes y otros tienden a oponerse y resistirse a tal dominación o hegemonía (p. 11). 
Una de las dimensiones del currículo es la dimensión ideológica, cuyo análisis puede hacerse a través del currículo oculto (De Alba, 1998), de gran interés aquí, y definido como el:

conjunto de normas y valores inconscientes de conducta, aprendidas en la primera infancia y perpetuadas después en la escuela mediante los contenidos y, sobre todo, a través de los comportamientos, actitudes, gestos y expectativas respecto de alumnas y alumnos. En muchos casos entra en contradicción con nuestras ideas conscientes, perpetuando de ese modo, conductas no deseadas de modo expreso (Spadaro 2008 - 2009, p. 130).

En otras palabras, en las instituciones educativas se promueven unos aprendizajes no intencionados, no previstos e incluso a contravía del mismo currículo explícito, como cuando el o la docente enseñan democracia siendo autoritarias/os en clase y por fuera de ella ante sus estudiantes. Es muy probable que las/os docentes, en nuestro afán de ceñirnos al currículo explícito, no cuestionemos presupuestos implícitos en el currículo oculto.

El currículo oculto permea todos los aspectos de la enseñanza, entre ellos los textos escolares. La importancia de analizarlos radica en que éstos son "mecanismos invisibles a través de los cuales aparecen y se refuerzan desigualdades” (Terrón y Cobano-Delgado, 2008, p. 387). Así, cuando se analiza el rol de las mujeres y de los varones en los textos escolares "podemos conocer no sólo las ideas, los valores o las actitudes latentes en una sociedad, sino además la forma en la que los conocimientos son transmitidos en las aulas tanto a niños como a niñas" (p. 387).

Se podría afirmar que los textos escolares reflejan los roles que en la sociedad han sido asignados a varones y mujeres. Y que los esfuerzos por incluir en tales textos el papel de las mujeres vinculado con profesiones distintas a la de ser amas de casa no es suficiente (Grinberg y Palermo, 2000). Por ello, y como afirma Spadaro (2008-2009), "resulta fundamental el análisis del proceso de construcción de la identidad femenina y masculina y aquello con lo que nos identificamos o desidentificamos unas y otros ya que dicho proceso de identificación es continuo y cambiante" (p. 130).

Desde la antigüedad, las mujeres han sido relegadas a oficios relacionados con el hogar y el cuidado de sus hijos (De Martino y Bruzzese, 2000). Y aún conservamos la idea de que las mujeres, así se desarrollen y triunfen profesionalmente, tienen un rol ineludible relacionado con aquellos quehaceres. En una investigación sobre el papel de las mujeres en las ilustraciones de los libros de texto de educación primaria, en España, las investigadoras concluyeron que, a pesar de los avances significativos (por ejemplo, en una mayor alusión de las mujeres), debe reconocerse las limitaciones que aún sostiene la población femenina, y que se manifiesta de algún modo en las siguientes conclusiones:

los espacios públicos están asociados a los hombres, mientras que los privados se relacionan con la mujer. (...) se siguen asociando con relativa frecuencia actitudes de alegría y felicidad a la figura femenina, no siendo tan habituales la de agresividad o enfado. (...) son más numerosas las profesiones referidas a los hombres que a las mujeres. (...) -aunque esté presente- la mujer sigue ocupando puestos secundarios. Curiosamente, es más fácil encontrar en los textos representaciones gráficas de hombres realizando tareas domésticas, que de mujeres trabajando en puestos de un mayor rango. (...) se aprecia un intento de equilibrar el número de hombres y mujeres, chicos y chicas en los dibujos. (...). Parece como si para afrontar el tratamiento de la cuestión de género se optara en multitud de ocasiones por representar figuras que podríamos denominar asexuadas, en las que es imposible determinar si el personaje representado es chico o chica (Terrón Caro y Cobano- Delgado, 2008, p. 399).

Terrón Caro y Cobano Delgado (2008) afirman que "ya antes de su incorporación a la escuela, la mayor parte de las conductas discriminatorias han sido aprendidas” (pp. 386-387) desde la familia, y la escuela se encarga de afianzar esos estereotipos (Subirats y Tomé, 1992). Y dado que como la ilustración puede ser lo que más llame la atención del niño o de la niña, se sugiere que ésta muestre situaciones en las que los varones realizan labores del hogar y las mujeres realizan sus deseos representados en éxito social o desarrollo profesional. La conclusión de Grinberg y Palermo (2000), es que no es suficiente con que los textos escolares incluyan o muestren el trabajo de las mujeres (en nuestro caso, el aporte intelectual de ellas), si tal incorporación no contribuye a una reflexión de los comportamientos concretos de varones y mujeres, en la que se evite "una naturalización de los roles asignados tradicionalmente a ambos sexos" (p. 213). De hecho, 
según esta investigación, en Argentina los textos escolares muestran cómo las mujeres se han incorporado al mundo laboral tradicionalmente masculino, mas enfatizando aún en que la responsabilidad del hogar sigue siendo de la mujer.

Cambiar los textos escolares por unos cuidadosamente estructurados en equidad de género no sería suficiente, dado que estos textos no son más que un mecanismo, entre otros, que la escuela mantiene vigente y a través de los cuales se ejerce la violencia simbólica contra las mujeres. Por ejemplo, Dubet (2011, pp. 37-40) describe cómo pequeñas desigualdades en la escuela se van sumando a otras hasta generar grandes desigualdades escolares. Es una prioridad, por lo tanto, identificar esas pequeñas desigualdades, visibilizarlas, confrontarlas e intentar eliminarlas:

Mecanismos idénticos obran contra las alumnas: mejores como estudiantes que los varones, pierden progresivamente esta
ventaja por la suma de las elecciones y el juego de las orientaciones hacia carreras y oficios menos rentables -sin que ninguna
de las etapas de su recorrido escolar haya sido brutalmente discriminatoria-. Desde los juegos con muñecas hasta supuestos
'gustos' literarios, pequeñas diferencias se acumulan, que a la postre se vuelven grandes desigualdades. (pp. 39-40) (...) Todo
esto es tan evidente que las mujeres mismas anticipan esta discriminación y tienen menos ambiciones que los hombres, aun
cuando su rendimiento académico sea generalmente mejor que el de los varones. Dicho de otro modo, no basta con haber
abierto el acceso a los lugares para crear la igualdad: ni los modelos culturales favorables a los hombres, ni la economía de la
vida familiar han sido profundamente afectados por esta aparente democratización (p. 47).

En efecto, la ausencia de las mujeres en el canon filosófico (algo que veremos en la siguiente sección) puede considerarse como una pequeña desigualdad que se va sumando a otras del dominio patriarcal que se manifiestan y refuerzan en la escuela.

Igualmente interesante es mostrar que incluso los varones que desean instaurar igualdad de condiciones entre varones y mujeres, no pueden hacerlo porque la estructura misma de la sociedad está construida sobre la base de una división de sexos de la que no solemos ser conscientes. Sin una comprensión de cómo funciona esta estructura todo esfuerzo podría ser en vano, puesto que se contribuye a que continúe imponiéndose la dominación masculina (Bourdieu, 2000).

Por otro lado, parece que no se ve bien que una mujer asuma roles que tradicionalmente han jugado los varones. Pues cuando las mujeres alcanzan lo que se les pide en el ideal de la educación, se las recrimina: "para ser una persona educada, una mujer tiene que adquirir rasgos que se aprecian negativamente cuando ella los posee" (Martin en: Davis, 1996, p. 529). La opinión de Bourdieu (2000) al respecto es que se observa que cuando una mujer es exitosa profesionalmente debe pagar ese éxito con un éxito menor en el orden doméstico, y viceversa; lo que refleja parte de la realidad de las relaciones estructurales de dominación sexual, que no son pocas ${ }^{6}$. Detener esta violencia simbólica contra las mujeres se convierte en una necesidad inaplazable. Irónicamente, esto sólo puede lograrse desde una de las instituciones que han contribuido a mantener esta violencia: la escuela (Van Dijk, 2010; Álvarez Uría, 1995). Las/osos docentes cargan con una responsabilidad intransferible y se convierten, así, en agentes transformadoras/es.

La situación problemática está planteada, y con ella la tarea. Tal y como afirma Bourdieu, en palabras de Galak (2014): "justamente la tarea de la o el cientista social es preguntarse por las lógicas de poder y de dominación simbólica que atraviesan el objeto de estudio y que fueron incorporadas en un largo proceso socio-histórico de apropiación" (p. 362).

En síntesis, la escuela se convierte en un mecanismo de reproducción de desigualdades y de violencia simbólica contra las mujeres.

\section{LA FILOSOFÍA COMO CREADORA Y REPRODUCTORA DE ESTEREOTIPOS PATRIARCALES}

(...) debido a que el género es excluido como categoría de análisis en la teoría educativa, las dicotomias sobre las que nuestra tradición filosófica descansa permanecen inadvertidas. Este currículo oculto de misoginia perpetúa la devaluación de lo femenino en el discurso y la marginalización de las mujeres y sus logros en el mundo tangible. (...). 
La omisión de mujeres filósofas como Mary Wollstonecraft y Mary Astell de las antologias y del currículo estándar importa, afirma Martin, porque transmite "el mensaje de que las mujeres no son capaces de reflexión filosófica significativa” (...). La invisibilidad de las mujeres en la filosofía de la educación perpetúa el statu quo patriarcal al mantener que los sexos son desiguales y que los logros de las chicas y las mujeres no son valiosos ni importantes (Martin, 1994, p. 45, en Davis, 1996, p.529).

Hay un buen número de filósofas identificadas en todos los períodos históricos de la filosofía (De Martino y Bruzzese, 2000). Se puede encontrar no pocos registros históricos en los que las mujeres son constructoras de saberes; por ejemplo, la compilación en cuatro tomos sobre filósofas organizada por Waithe $(1987,1989$, 1991, 1995) es contundente en este asunto, aunque allí no reconozca a filósofas hispanoparlantes ${ }^{7}$.

Sin embargo, el canon filosófico no incluye a las mujeres (Davis, 1996; Kalnická, 2010; Waithe, 2015). Los textos escolares con los que se enseña filosofía en la escuela, y los libros de filósofos con los que se trabaja en la universidad para formar a las/os que enseñan filosofía, en consecuencia, referencian en su mayoría a varones (Terrón Caro y Cobano-Delgado, 2008; Grinberg y Palermo, 2000; Alzate, Gómez, y Romero, 1999; entre otros). Y si el currículo explícito no las incluye, ¿qué podríamos encontrar en un análisis del currículo oculto? Podría pensarse que ese problema no es tal, y no tiene sentido abordarlo hoy, cuando se tiene un buen número de filósofas incorporándose al canon como Hannah Arendt, Judith Butler, Adela Cortina, entre otras, sin la oposición y la dificultad del pasado debido a circunstancias históricas determinadas que ya no están. Sin embargo y como veremos, el problema va más allá de la ausencia de las mujeres en el canon.

Según Bourdieu (2000), la escuela continúa "transmitiendo los presupuestos de la representación patriarcal” (p. 63). Es interesante el análisis que este autor hace de la institución escolar porque visibiliza el uso de modelos arcaicos como la tradición aristotélica que divide lo masculino y lo femenino al hacer "del hombre el principio activo y de la mujer el elemento pasivo" (p.63) y cómo esto desemboca en que:

Los hombres siguen dominando el espacio público y el campo del poder (especialmente económico, sobre la producción) mientras que las mujeres permanecen entregadas (de manera predominante) al espacio privado (doméstico, espacio de la reproducción), donde se perpetúa la lógica de la economía de los bienes simbólicos, o en aquellos tipos de extensiones de ese espacio llamados servicios sociales (hospitalarios especialmente) y educativos o también en los universos de producción simbólica (espacio literario, artístico o periodístico, etc.) (p. 68).

Esta conclusión de la filosofía aristotélica no es aislada. El ideal moderno de la filosofía da supremacía a la razón por encima de cualquier otra posibilidad o forma de llegar al saber. Con el Yo pienso, luego existo cartesiano quedan por fuera la emoción y el sentimiento, elementos que han sido adjudicados erróneamente a la femineidad, como si no fuera propio de varones y mujeres. Adicionalmente, filósofas feministas que han estudiado el canon concluyen que la ausencia de las mujeres en él es generalizada. Por lo que se acusa a la filosofía de androcéntrica y misógina (Witt y Shapiro, 2017).

Ante la pregunta, ¿cómo educar de la mejor manera a niñas y mujeres en un mundo que es dominado por el androcentrismo y la misoginia?, existen unas respuestas valiosas, aunque parciales, recogidas en dos textos cuya reseña hace Hilary Davis $(1996)^{8}$, y que muestran la complejidad del problema; por ejemplo, la misma división cartesiana de cuerpo y mente ya lleva consigo un discurso androcéntrico. Jane Roland Martin se aproxima a tal dicotomía "desde una perspectiva filosófica, al examinar el discurso dominante de la filosofía de la educación, su sesgo androcéntrico, y las contradicciones que las mujeres enfrentan en un ambiente académico que idealiza las mentes incorpóreas (masculinas)" (Davis, 1996, p. 525). Sin embargo, ¿đde qué modo la separación cartesiana mente-cuerpo puede constituir una amenaza para las jóvenes en la escuela? Martin afirma que ese marco metafísico necesariamente genera "mentes incorpóreas y cuerpos dóciles", y constituye nada menos que una de las "dicotomías jerárquicas fundacionales de la filosofía occidental que asocia al hombre con las categorías dominantes de razón, mente y cultura mientras devalúa las categorías asociadas exclusivamente con lo femenino: emoción, cuerpo y naturaleza" (Martin en: Davis, 1996, p. 526). Por lo que esas "metáforas vinculadas al dualismo mente-cuerpo no son meros patrones de pensamiento, sino que tienen consecuencias reales para las mujeres (y para los varones) en el mundo tangible” (Davis, 1996, p. 526 ). Lo que tiene como consecuencia que, 
para ser educadas [las mujeres] deben renunciar a su propio modo de experimentar y mirar al mundo, enajenándose de sí mismas. Para evitar la enajenación, tienen que permanecer sin educación. Además, para ser una persona educada, una mujer tiene que adquirir rasgos que se aprecian negativamente cuando ella los posee. Al mismo tiempo, los rasgos que son evaluados positivamente cuando son poseídos por ella -por ejemplo, ser compasiva y empática- son excluidos del ideal. (Martin en Davis, 1996, p. 529)

De algún modo, el ideal de la educación le pide a las mujeres que se violenten a sí mismas: "Y dado que las mujeres 'son los otros incorporados, a los que no se les permite no tener un cuerpo', la mujer que piensa es una anomalía que amenaza y transgrede los límites del pensamiento dualista. Ella se percibe como disruptiva y revoltosa, incluso no natural a su sexo" (Davis, 1996, p. 529). Es decir, al presuponer que una mujer no debe pensar, cuando lo hace "pierde" lo que la caracteriza biológicamente como mujer. No obstante, desde ese marco, no alcanza a ser varón, y ya ha perdido algo de su femineidad; es un individuo raro. Ese supuesto se transmite desde el currículo oculto, por lo que la escuela, desde una enseñanza de la filosofía sin perspectiva de género, fomenta los valores de la racionalidad moderna, androcéntrica. Ello no significa que las mujeres deban reclamar la especificadad de su cuerpo, porque ello acarrearía una defensa de la relación binaria hombre-mujer sustentada en un biologicismo que debe superarse aún en la sociedad.

\section{El rol del y la docente y la escuela, al respecto de la violencia simbólica contra las mujeres}

La formación docente en filosofía deberia "formar", básicamente, a alguien que esté
en condiciones de resolver el problema de enseñar filosofía, en situaciones diversas. No
a alguien que tenga meramente "herramientas"para enseñar, sino a alguien que sea
capaz de evaluar los supuestos que acompañan las distintas herramientas (filosóficos y
pedagógicos, pero también sociales, de género, culturales, etc.) y por qué fueron así
diseñadas, con qué objeto, con qué sentido. Esto permitirá que los futuros profesores y
profesoras estén en mejores condiciones de elegir sus métodos y recursos para enseñar,
en consonancia con su compromiso con la filosofía y la educación.

(Cerletti, 2008, p. 49)

A pesar de lo anterior, la escuela también representa el lugar donde se pueden corregir estas desigualdades a través de la formación ciudadana. Hay una serie de acciones concretas que se pueden llevar a cabo por parte de docentes. El punto inicial ineludible es empezar a incluir filósofas en el canon. No es necesario excluir a los filósofos ni mucho menos excluir los pasajes de misoginia. Por el contrario, sirven de insumo para el análisis que debe haber en las aulas de clase: "Descubrir el sexismo a través de la lectura de fuentes es un ejercicio por demás enriquecedor e incluso divertido" (Spadaro, 2008 - 2009, pp. 132-133). No es necesario esperar a que la academia haga la inclusión de algunas filósofas en el canon, porque él o la profesional en el área tiene las herramientas suficientes para identificar cualquiera pensadora de acuerdo a sus intenciones pedagógicas, y hay información en la web con listas de más de doscientas filósofas identificadas (para dar sólo un ejemplo, véase el sitio web de laSociety for the Study of Women Philosophers (2017) para encontrar un centenar de ellas.

¿Qué ganamos con incluir en el canon a las filósofas si, como ya hemos dicho, no es suficiente? Inicialmente, siguiendo a Dykeman (en Kalnická, 2010), ayuda a suministrarles a nuestros/as estudiantes una imagen más apropiada de la historia de la filosofía y del desarrollo de cuestiones filosóficas; de acuerdo con Gardner (en Kalnická, 2010), ofrece soluciones que pueden cambiar nuestro punto de vista filosófico contemporáneo, especialmente en la filosofía moral; para Bordo (en Kalnická, 2010), la inclusión de filósofas en el canon tradicional ayuda a comprender qué excluye y qué silencia. Spadaro (2008-2009) cree que una de las acciones es formar al profesorado, pues al no estar actualizado con las nuevas investigaciones sobre el género en la filosofía, cree que está siendo igualitario cuando lo que hace es reproducir el estereotipo androcéntrico, incluso rechazando la propuesta por considerarla "feminista" y sectaria (p. 131). El o la docente corre el riesgo de no ver en lo naturalizado algo que deba ser corregido, de no ver que en el ejercicio pedagógico ya hay implícito un modo de dominación que reproduce sin percatarse: "la (di)visión del mundo que transmite toda 
educación (...) encierra en su interior una manera naturalizada de dominación, que no sólo repercute en la diferencia biológica sino que genera una distinción social” (Galak, 2015, pp.138-139).

Adicionalmente, el o la docente corre un riesgo, que comparte con las/os demás adultas/os, y es que cuando son figuras admiradas, influencian más fácilmente a los chicos y chicas. Y esa influencia transmite esos modos de dominación masculina. En palabras de Mauss (1997, p. 389), se trata del prestigio de la persona que realiza la acción ordenada que imita el niño o la niña de quienes han tenido éxito. Este éxito hace referencia a la eficacia del instrumento a través del cual se transmite la cultura, y que Mauss define como técnicas del cuerpo (p. 391). Estas técnicas son adquiridas para toda la vida en la etapa de iniciación, mas el momento decisivo es el de la adolescencia, lo que hace central la intervención del currículo y la formación docente en equidad de género, en la educación secundaria principalmente. Por ello, Spadaro (2008 - 2009) sugiere que las/os docentes hagan un trabajo crítico. No hay que cambiar los textos filosóficos: "Debe desarrollar una estrategia crítica: contextualizarlos, situarlos. Debe hacer muchas preguntas. ¿De dónde surgen? ¿Cómo surgen? ¿En interlocución con quién? ¿En qué contextos reales, personales y sociales, el o la filósofa pensó lo que pensó? ¿Es cierto que dicho contexto histórico no permitía pensar de otra manera? Intentar responder a estas cuestiones nos permite encontrar nuevos caminos en la misma filosofía" (pp. 133-134). Adicionalmente, sugiere que revisen los contenidos: "Podemos elegir contenidos más tradicionales u orientar la elección hacia una óptica de análisis e interpretación no androcéntrica, favoreciendo así la igualdad de oportunidades de los sexos y permitiendo el protagonismo de múltiples voces no hegemónicas" (pp. 132).

Siguiendo a Puleo (en Spadaro, 2008 - 2009), las/os docentes deberían:

Releer los clásicos del pensamiento como ejercicio ineludible, y por demás divertido, para una comprensión profunda de las relaciones sociales entre los sexos. (...) Colaborar en la constitución de un corpus filosófico no sexista, rastreando en el pasado una línea filosófica emancipatoria perdida. Recuperar la voz de las y los olvidados permite comprender mejor el pensamiento de los filósofos estudiados en la tradición hegemónica. (...) Reconocer la producción de las filósofas, pues en pocos terrenos es tan absolutamente clara la ausencia de reconocimiento como en el ámbito filosófico, por ser casi el más impropio para las mujeres. (...) Incluir la creación filosófica que surge en los debates internos de la crítica feminista. (p.135)

Campagnoli (2015) propone "abarcar cuestiones no sólo de contenido sino también organizacionales, aplicables a todas las estructuras de la docencia; es decir, educación con niñas/os, jóvenes y adultas/os” (p. 100), y algunas sugerencias sobre la selección de estrategias y contenidos. Algunas de ellas serían: revisar los sistemas de comunicación para evitar el lenguaje sexista, estar atentas/os a que las/osestudiantes disfruten del espacio equitativamente, por ejemplo en el patio, velar porque los recursos didácticos sean utilzados equilibradamente por las/os jóvenes, rechazar materiales sexistas, entre otras.

Por su parte, Da Cunha (2015) propone reformular algunos de los temas de los programas de filosofía y psicología desde una perspectiva de género.Nosotros proponemos, además de acoger todas las sugerencias anteriores, incluir en nuestra clases una perspectiva en equidad de género (que difícilmente se da en Colombia), y para ello qué mejor que comenzar incluyendo en el plan de estudios el pensamiento de filósofas latinoamericanas como Francesca Gargallo, Silvia Rivera Cusicanqui, Ivone Gebara, Ofelia Schutte, Graciela Hierro Perezcastro, Eli Bartra Murià, Diana Helena Maffia, María Luisa Femenías, Urania Ungo, María del Rayo Ramírez Fierro, Rosario Castellanos, María Lugones, entre otras. Con ello no sólo devaluaríamos un eurocentrismo a favor de un pensamiento propio, vital, descolonizador, sino que visibilizaríamos mecanismos de reproducción de desigualdades en un auténtico ejercicio filosófico que, de lejos, es más deseable que discutir en la academia las "mejores" interpretaciones de un pensador canónico.

La escuela tiene esa responsabilidad en visibilizar la violencia simbólica contra las mujeres y los mecanismos de reproducción de la misma. Eso no significa que la escuela tenga la responsabilidad de eliminar la inequidad de género, no sólo porque eso la desborda sino porque le compete a otras instancias políticas. Significa que la escuela no debe dejar de pensarse y evolucionar en sus reflexiones, que debe evaluarse constantemente. Para Cerletti (2008), el o la docente de filosofía tiene que tener las herramientas para enseñar, no obstante también estar en capacidad "de evaluar los supuestos que acompañan las distintas herramientas (filosóficos y 
pedagógicos, pero también sociales, de género, culturales, etc.) y por qué fueron así diseñadas, con qué objeto, con qué sentido" (p. 49). De lo contrario, el docente parecería más un agente "entrenado" para mantener el control derivado del currículo, como denuncian Masschelein y Simons (2014, p. 69), que un/amaestro/a que ame su asignatura, a sus estudiantes, y que genera interés en ellas/os derivado de su compromiso.

Finalmente, y como conclusión, debe decirse que la ausencia de las mujeres en el canon filosófico es tanto una manifestación como un mecanismo del poder patriarcal. Que la escuela debe visibilizar esa ausencia porque eso contribuye a la lucha contra la inequidad que ella misma reproduce. Y una manera de hacerlo es a través de un currículo más inclusivo donde sea visto como natural que el varón también pueda ocuparse de labores domésticas y que las mujeres reflexionan y pueden ocupar un papel igual de trascendental en la vida pública.

El análisis de las consecuencias pedagógicas en la formación de nuestras/osestudiantes se hace más urgente y necesario una vez se descubre las particularidades de este problema. En efecto, es un problema que merece toda nuestra atención, y ante el cual, como docentes de filosofía, no podemos estar indiferentes. Habrá otras reflexiones que nos lleven a sugerir otras acciones. Sin embargo, ninguna de ellas sería posible, quizás, sin aceptar que hay un problema. Un problema que pretendía pasar desapercibido por la supuesta neutralidad del ejercicio filosófico.

\section{Referencias bibliográficas}

Álvarez Uría, F. (1995). Escuela y subjetividad. Recuperado de: https://www.fceia.unr.edu.ar/geii/maestria/DoraBib liografia/Ampliatoria/Alvarez\%20Ur\%C3\%ACa.pdf

Alzate Piedrahita, M. V., Gómez Mendoza, M. Á., y Romero Loaiza, F. (1999). Textos Escolares y Representaciones Sociales de la Familia. III. Representaciones de la familia en los textos de ciencias sociales de la educación básica primaria. Dosquebradas: Botero Gómez y Cía. Ltda

Amorós, C. (1985). Hacia una crítica de la razón patriarcal. Barcelona: Anthropos

Bach, A. M. (2015). Género, estereotipos y otras discriminaciones como puntos ciegos. En Bach, A. M. (Ed.). Para una didáctica con perspectiva de género (pp. 15-58). Buenos Aires: Miño y Dávila Editores

Bourdieu, P. (2000). La dominación masculina. Barcelona: Anagrama.

Bourdieu, P. y Saint Martin, M. (1998). Las categorías del juicio profesoral. Propuesta educativa, 19, 4-18

Campagnoli, M. A. (2015). ¡Andá a lavar los platos! Androcentrismo y sexismo en el lenguaje. En Bach, A. M. (Ed.). Para una didáctica con perspectiva de género (pp. 59-106). Buenos Aires: Miño y Dávila Editores

Cerletti, A. (2008). La enseñanza de la filosofía como problema filosófico. Buenos Aires: Libros del Zorzal

Da Cunha, M. (2015). El currículum como Speculum. En Bach, A. M. (Ed.).Para una didáctica con perspectiva de género (pp. 153-209). Buenos Aires: Miño y Dávila Editores

Davis, H. E. (1996). Docile Bodies and Disembodied Minds. [Revisión del libro Changing the Educational Landscape: Philosophy, Women, and Curriculum, por J. R. Martin]. Educational Theory, 26 (4), 525-543. Recuperado de h ttps://doi.org/10.1111/j.1741-5446.1996.00525.x

De Alba, A. (1998). Las perspectivas. En De Alba, A. Currículum: crisis, mito y perspectivas. Buenos Aires: Miño y Dávila Editores. Recuperado de: http://www.terras.edu.ar/biblioteca/1/CRRM_De_Alba_Unidad_1.pdf

De Martino, G.; Bruzzese, M. (2000). Las filósofas. Las mujeres protagonistas en la historia del pensamiento. Madrid: Ediciones Cátedra

Dubet, F. (2011). Repensar la justicia social. Contra el mito de la igualdad de las oportunidades. Buenos Aires: Siglo XXI Editores

Femenías, M. L. (1996). Inferioridad y exclusión: un modelo para desarmar. Buenos Aires: GEL

Femenías, M. L. (2008). Violencia contra las mujeres: urdimbres que marcan la trama. En Aponte Sánchez, É. y Femenías, M. L. (comp.). Articulaciones sobre la violencia contra las mujeres (pp. 13-53). La Plata: Universidad Nacional de La Plata 
Galak, E. (2014). Construir el cuerpo: cuatro consideraciones epistemo-metodológicas y tres metáforas para pensar el objeto de estudio "cuerpo". Poiésis, 8 (14), 348-364. doi: http://dx.doi.org/10.19177/prppge.v8e14201434 $8-364$

Galak, E. (2015). Esbozos de una teoría de la práctica de educar. Pierre Bourdieu, educación de los cuerpos, violencia y capital simbólico. Revista Tempos e Espaços em Educação, 8 (15), 133-144. doi: https://doi.org/10.20952/rev tee.v8i15.3669

Gargallo, F. (2006). Las ideas feministas latinoamericanas. México: UACM

Grinberg, S. y Palermo, A. (2000). Mujeres y trabajo en los textos escolares: crisis y perspectivas. Educere, 4 (11), 207-214

Kalnická, Z. (2010). Dislocating Women from Philosophy: Five Strategies. Clepsydra, 9, 143-157

Lerner, G. (1990). La creación del patriarcado. Barcelona: Crítica

Martin, J. R. (Ed.). (1994). Changing the Educational Landscape: Philosophy, Women, and Curriculum, New York: Routledge

Masschelein, J., y Simons, M. (2014). Defensa de la escuela. Una cuestión pública. Buenos Aires: Miño y Dávila Editores Mauss, M. (1997). Las técnicas del cuerpo. En J. Crary, y S. Kwinter (ed.), Incorporaciones. Madrid: Cátedra

Millett, K. (1995). Política sexual. Madrid: Ediciones Cátedra. Universitat de València

Pateman, C. (1995). El contrato sexual. Barcelona: Anthropos

Society for the Study of Women Philosophers, Inc. (2017). Recuperado de http://www.societyforthestudyofwomen philosophers.org/Women_Philosophers_Start.html

Spadaro, M. C. y Femenías, M. L. (comp.) (2012). Enseñar filosofía, hoy. La Plata: Universidad Nacional de La Plata

Spadaro, M. C. (noviembre - abril de 2008 - 2009). Respuestas no hegemónicas a los problemas de la inclusión del género en la enseñanza de la filosofía: enfrentar el problema desde todos lados. Revista Sul-Americana de Filosofia e Educação - RESAFE, 11, 126-138

Subirats, M. y Tomé, A. (1992): La educación de niños y niñas. Recomendaciones institucionales y marco legal. Barcelona: Bellaterra

Terrón Caro, M. T. y Cobano-Delgado Palma, V. (2008). El papel de la mujer en las ilustraciones de los libros de texto de educación primaria. Revista Foro de Educación, 10, 385-400

Valcárcel, A. (1991). Sexo y Filosofía. Sobre "mujer" y "poder". Barcelona: Anthropos.

Van Dijk, T. A. (2010). Análisis del discurso del racismo. Crítica y emancipación, 3, 65-94. Recuperado de: http://bi blioteca.clacso.edu.ar/ojs/index.php/critica/article/view/168

Waithe, M. E. (ed.). (1987). A History of Women Philosophers. Ancient Women Philosophers, 600 B.C. - 500 A.D. (Vol. I). Dordrecht: Martinus Nijhoff Publishers

Waithe, M. E. (ed.). (1989). A History of Women Philosophers. Medieval, Renaissance, and Enlightenment (Vol. II). Dordrecht: Kluwer Academic Publishers

Waithe, M. E. (ed.). (1991). A History of Women Philosophers. Modern Women Philosophers, 1600 -1900 (Vol. III). Dordrecht: Springer Science - Business Media, B.V.

Waithe, M. E. (ed.). (1995). A History of Women Philosophers. Contemporary Women Philosophers, 1900 - today (Vol. IV). Dordrecht: Springer Science - Business Media, B.V.

Waithe, M. E. (2015). From Canon Fodder to Canon-Formation: How Do We Get There from Here? The Monist, $98,21-33$

Wieviorka, M. (2006). La violencia: destrucción y constitución del sujeto. Espacio abierto, 15 (1 y 2),239-248

Witt, C., y Shapiro, L. (2017). Feminist History of Philosophy. Stanford Encyclopedia of Philosophy. Recuperado de: h ttps://plato.stanford.edu/entries/feminism-femhist/ 


\section{Notas}

1. Aponte y Femenías (2008, p. 27) afirman que el primer análisis sistemático y técnico del concepto de "dominación masculina” fue hecho por Kate Millet en su Politica sexual (1995) de 1969. Cito a Bourdieu porque me interesa mostrar su concepto de lógica de la denegación aplicado en la escuela, sin menoscabo de las descripciones y análisis de otras filósofas que han pensado el problema del patriarcado, como Amorós (1985), Lerner (1990), Pateman (1995), Valcárcel (1991) y Femenías (1996), aunque la lista es mayor, según Campagnoli (2015, p. 60).

2. Comprendemos el riesgo de usar la categoría "mujer" tanto en singular como en plural. Como Butler señala (parafraseada por Da Cunha, 2015), se trata de un sistema de definición que se convierte en un dispositivo de exclusión, al reducir al individuo nombrado a sus características biológicas, puesto que si una mujer es quien tiene vagina, entonces las travestis no serían mujeres, y no solo eso: incluso si se pretende defender a quien es definida como mujer, quien no se ajuste a esa definición sería negada, no reconocida o vista como alguien con algo que debe ser corregido. En otras palabras, acudir a la categoría mujer excluye a otros modos desde los que se puede ser mujer, como etnia, clase, sexo, religión o edad, entre otros, y estos modos no son fijos o constantes. Sin embargo, hay algo más revelador, que cuando una persona nace hembra y sobre su biología se ponen (como si fueran adornos) las características que el patriarcado ha dado a la mujer, se muestra que no sólo el género sino también el sexo son construcciones culturales, tal como Beauvoir afirmaba: una no nace mujer, sino que se hace. Podría decirse con Butler: la hacen con el discurso performativo, lo que se evidencia en la escuela cuando las y los docentes exigen a las niñas que se sienten de tal modo y no de otro, o que no jueguen bruscamente, etc. Así, cuando apelamos a la categoría "mujer", hacemos referencia no al individuo de sexo biológico femenino, sino al individuo que es distinta/o de la posición dominante patriarcal, androcéntrica, sin que por ello caiga necesariamente en el binarismo que se sugiere deconstruir, puesto que de dos géneros fijos reconocidos por el patriarcado se pasa al reconocimiento de variadas e inestables formas de ser y estar.

3. Una aproximación similar la expone Campagnoli (2015) al definir androcentrismo como "una perspectiva que legitima [la producción del significado de varón dominante] a la vez que la esconde” (pág. 75).

4. Neutralidad, en la medida en que se esperaría que una disciplina que usa el razonamiento lógico, que previene el uso de las falacias, que busca la verdad (aunque no haya consenso sobre la posibilidad de alcanzarla), que interpela a las instituciones y a la sociedad en busca de argumentos que justifiquen su actuar ético, que evalúe tales argumentos, que denuncie cómo funcionan los mecanismos de poder, entre otros asuntos... se esperaría que la filosofía no violentara a las mujeres. Lo cierto es que desde el inicio, el ejercicio del pensamiento no es para todos ni para todas, y que la palabra "hombre" o "ser humano" sólo hace referencia al varón libre y con recursos económicos (no se piensa con hambre), mas no a los individuos pobres, esclavos, niños ni, mucho menos, a mujeres.

5. Entiéndase por currículum nulo el "conformado por aquellos saberes que son excluidos de los contenidos 'oficiales' de la enseñanza” (Da Cunha, 2015, p. 156).

6. El patriarcado ejerce violencia simbólica contra las mujeres desde el androcentrismo y desde el sexismo, y lo hace de múltiples formas. Campagnoli (2015) aclara que el androcentrismo es un punto de vista que pone al varón en el centro y a lo no varonil en la periferia, y el sexismo es el menosprecio de las mujeres y/o a identidades sexo-genéricas distintas a la identidad masculina. Desde el androcentrismo, se expresa en la opacidad androcéntrica del discurso es cuando se hace equivaler hombre a humano o universal o neutral, como si realmente incluyera lo que no incluye: a las mujeres $\mathrm{u}$ otras colectividades. Tal es el caso de la Declaración Universal de los Derechos del Hombre y el Ciudadano de 1789 , que ni es universal ni incluye a la mujer, por lo que Olympe De Gouges se atrevió a publicar la Declaración Universal de los Derechos de la Mujer y la Ciudadana (por ello asesinada, en un ejemplo de cómo se puede transformar la violencia simbólica a violencia física explícita). Otro ejemplo es la Ginopia: cuando la cultura no ve ni escucha a las mujeres. Por otra parte, se expresa en el lenguaje al usar el masculino generalizante, y en las fuertes resistencias a usos alternativos a la utilización del masculino generalizante. Desde el sexismo se manifiesta en la sexualización, al dar demasiada importancia a la apariencia de las mujeres y no a su intelecto, o con la erotización de la dominación, concepto de Gerda Lerner definido por Campagnoli (2015) como: "un modo de mostrar como preferida por la mujer la imposición que realiza el varón desde su propia voluntad y/o deseo” (p. 89); es decir, mostrar a las mujeres complacientes en la condición de subordinadas; mecanismo recurrente en el humor de corte sexista, cuyos chiste "no sólo niega los prejuicios en que se asientan, sino que refuerzan las diferencias y reproducen las relaciones de desigualdad” (pág. 99). En segundo lugar se expresa a través de la misoginia, por ejemplo en el caso del piropo, pues el deseo masculino heterosexual establece a la mujer como una propiedad de los hombres. Otros ejemplos son la feminización de los hombres como estrategia de humillación y las fobias sexuales, que no aceptan otra relación distinta a la heterosexual.

Es frecuente en nuestra experiencia como docente de escuela escuchar cada una de esas manifestaciones contras las mujeres por parte de estudiantes y adultas/o. Parece increíble que tal lista, por lo demás incompleta, no tenga una mayor visibilidad, y que muchas mujeres, incluso formadas profesionalmente, no estén en capacidad de ver lo que sistemática y hábilmente el patriarcado ha ocultado. La perspectiva que nos interesa aquí es la opacidad androcéntrica del discurso, 
especialmente la ginopia que también se manifiesta con la exclusión de las mujeres filósofas del canon o del currículo de filosofía en la escuela; lo que también se denomina "violencia epistémica" (Da Cunha, 2015, p. 154).

7. El reconocimiento a nuestras filósofas debe iniciar desde nosotras/os mismas/os, por lo que no se explica que la filósofa cubana Ofelia Schutte no cite a teóricas feministas latinoamericanas en sus escritos para público norteamericano a pesar de sus relaciones académicas con la región, como reclama Gargallo (2006).

8. Para evitar esta pregunta de corte sexista de Martin, podríamos reformularla así: ¿cómo educar de la mejor manera a niñas y mujeres (también y especialmente a niños y varones) en un mundo que es dominado por el androcentrismo y la misoginia? 